\title{
Cardiovascular Outcomes and Angiotensin Converting Enzyme Inhibitors: Beyond Blood Pressure Control
}

\author{
Editorial to: "Secondary Prevention of Coronary Disease with ACE Inhibition- \\ Does Blood Pressure Reduction with Perindopril Explain the Benefits in EUROPA?" \\ by Remme et al.
}

\author{
Anil Verma • Hector O. Ventura \\ Published online: 13 November 2008 \\ (C) Springer Science + Business Media, LLC 2008
}

\begin{abstract}
Key words The renin-angiotensin system . Angiotensin-converting enzyme inhibitor · Hypertension . Atherosclerosis - Coronary artery disease . The Heart Outcome Prevention and Evaluation (HOPE) study . Perindopril in Stable Coronary Artery Disease (EUROPA)
\end{abstract}

Atherosclerosis and its complications, stroke, coronary artery disease and peripheral arterial disease, remain the leading cause of mortality and morbidity and are increasing in incidence in the developing world [1]. Multiple mechanisms are associated with the development of atherosclerosis but since the discovery of renin by Tigerstedt and Bergman [2] more that 100 years ago, the renin-angiotensin system (RAS) has been focus of intensive investigative efforts. Although our understanding of the RAS and the development of atherosclerosis has grown increasingly complex, inhibition of the RAS with an angiotensinconverting enzyme inhibitor (ACE-I) has become a firmly established therapeutic approach for reducing morbidity and the risk of death across a broad spectrum of cardiovascular diseases based on multiple, well-conducted, randomized clinical outcome trials (RCT) [3-6]. The benefits of ACE-I on clinical outcomes are due in part to

\footnotetext{
A. Verma $\cdot$ H. O. Ventura

Ochsner Heart and Vascular Institute,

New Orleans, LA, USA

A. Verma $\cdot$ H. O. Ventura $(\bowtie)$

Ochsner Medical Center,

1514 Jefferson Highway,

New Orleans, LA 70121-2483, USA

e-mail: hventura@ochsner.org
}

blood pressure control but other mechanisms beyond blood pressure lowering, such as their anti-atherosclerotic properties has been postulated in recent clinical trials [7]. These clinical trials have broadened our knowledge of management of cardiovascular risk

The Heart Outcome Prevention and Evaluation (HOPE) study [8] demonstrated the benefits of ACE-I on clinical outcomes in patients with established atherosclerosis or diabetes. The HOPE study by excluding patients with left ventricular systolic dysfunction and heart failure made obvious that the ACE-I ramipril not only reduced cardiovascular mortality, but also significantly reduced incidence of myocardial infarction and stroke. Similarly, the European Reduction of Cardiac Events with Perindopril in Stable Coronary Artery Disease (EUROPA) [9] study demonstrated that inhibition of the RAS with an ACE-I perindopril will result in secondary prevention of coronary events in a much broader population, patients with stable coronary disease irrespective of risk profile. The ACE-I perindopril in EUROPA [9] significantly reduced the combined endpoint of cardiovascular death, non-fatal myocardial infarction, and resuscitated sudden cardiac death by $20 \%$. However studies such as PEACE [10] failed to replicate the results of EUROPA and HOPE. In the PEACE trial, patients with coronary artery disease and with preserved left ventricular systolic function treated with ACE-I trandolapril in a dose of $4 \mathrm{mg}$ for several years did not show any reduction in cardiovascular outcomes. The lack of benefits of trandolapril in PEACE may be due to a lower cardiovascular risk population included in the trial and that patients were optimally treated with other evidence based treatments such as statins, beta blockers, antiplatelets and revascularization therefore they do not benefit with additional ACE-I. 
In this issue of the journal, Remme et al [11]. report the relationship between blood pressure lowering and the reduction in cardiovascular death, nonfatal myocardial infarction, or resuscitated cardiac arrest in a post hoc analysis of patients enrolled in EUROPA. The authors conclude that after about 4 years of follow up there was a significant reduction in the primary endpoint in patients on perindopril treatment compared to placebo with greatest relative risk reduction of $32 \%$ in the primary end point in patients with lowest blood pressure group $(<120 \mathrm{mmHg})$ in whom the study medication did not reduce blood pressure. Moreover the relative risk reduction on the primary endpoint during blinded treatment was comparable, irrespective of whether BP decreased or not or of the extent of BP reduction during perindopril treatment and no effect modification could be demonstrated of baseline systolic blood pressure level. Thus, the overall cardiovascular risk benefit of the perindopril treatment in EUROPA cannot be fully explained by baseline blood pressure or blood pressure reduction. The study by Remme et al. is important as it firms our belief in the beneficial effects of ACE-I irrespective of their favorable impact on blood pressure.

Angiotensin II - the end product of the renin-angiotensin system has an important effect on vascular structure and function and promotes vascular growth, smooth muscle cell migration, apoptosis, endothelial dysfunction, platelet aggregation, thrombosis, left ventricular hypertrophy, myocardial and vascular wall fibrosis, myocardial remodeling and thereby contributes to the development of hypertension, heart failure, and myocardial infarction [12]. In adult tissues, virtually all known deleterious effects of angiotensin II are attributable to the AT1 receptor. Production of pro-inflammatory cytokines, such as interleukin 1 , tumor necrosis factor $\alpha$, and especially interleukin 6, play a major part in the pathogenesis of atherosclerosis [13]. Both interleukin 6 and AT1 receptors have been detected in stable and unstable atherosclerotic plaques and there is evidence for a bidirectional crosstalki.e., interleukin 6 induced up-regulation of vascular AT1 receptor expression [14]. The adverse cerebral and cardiovascular effects of angiotensin II, which have potentially lethal sequelae, are pervasive. ACE-I reduce the production of angiotensin II and interfere with the pathophysiology of coronary ischaemia and renal insufficiency. Blockade of the RAS with ACE-I also increases bradykinin which in turn increases nitric oxide and prostacyclin production and thereby reduces oxidative stress. Bradykinin also increases tPA and thereby improves fibrinolytic balance, and has antiremodelling effects. Generation of the reactive oxygen species promotes and sustains the atherosclerotic process and activation of the matrix metalloproteinases through the activation of angiotensin II in the fibrous cap of the atherosclerotic lesion plays a role in plaque rupture. ACE-I decrease the expression of several adhesion molecules and play an important role in stabilization of the atherosclerotic plaque11.

Impaired endothelial function plays an important part in increased cardiovascular risk and angiotensin II has been shown to initiate and sustain several mechanisms that contribute to impaired endothelial function [15]. The Trial on Reversing ENdothelial Dysfunction [16] (TREND) study showed that ACE inhibition in patients with coronary artery disease improves endothelial function and several other studies in animal models and patients with coronary artery disease or hypertension have provided conclusive data on the favorable impact of ACE inhibition on anti-atherosclerotic effects, improvement in endothelial function and restoration of the fibrinolytic balance [17-19]. These effects are specific to ACE-I and are independent of blood pressure lowering and much more pronounced compared to beta-blockers, calcium antagonists and angiotensin receptor blockers [20].

The results of the PEACE added speculation that not all patients with coronary artery disease should receive ACE-I for secondary prevention [21]. Several explanations have been put forward to justify this discrepancy including a lower cardiovascular risk profile of the PEACE study population, which was more intensively treated with lipid lowering drugs and myocardial revascularization prior to enrollment than patients in EUROPA. However, subsequent analysis of the EUROPA study have shown that risk level had no impact on the beneficial effects derived from the perindopril treatment and the greatest favorable effects were seen in the medium risk population and in patients with a normal left ventricular systolic function [7, 22]. Moreover, these favorable effects of ACE-I are not beatable by angiotensin receptor blocker. The recently concluded ONTARGET [23] and the VALIANT [24] studies show that angiotensin receptor blockers telmisartan and valsartan provide a benefit similar to but not superior to that of a proven ACE-I. However, because of the increased cost associated with angiotensin receptor blockers and side effects, their primary value is as an alternative for patients who cannot tolerate ACE-I because of cough.

The central question for the clinician is whether the cardiovascular risk reduction can be achieved by mere lowering of the blood pressure. We do believe that the current work of Remme et al. provides more evidence that even after accounting for blood pressure changes over time, perindopril has a very significant impact in cardiovascular outcomes including patients with blood pressures lower than $120 \mathrm{mmHg}$. Although blood pressure control is paramount in decreasing cardiovascular risk, clinicians should be aware that other properties beyond blood pressure lowering are involved in improving clinical outcomes in patients with cardiovascular disease. 


\section{References}

1. Callow AD. Cardiovascular diseases 2005-the global picture. Vascul Pharmacol 2006;45:302-7.

2. Tigerstedt R, Bergman PG. Niere und Kreislauf. Skand Arch Physiol 1898;8:223-71.

3. Pfeffer MA, Braunwald E, Moye LA, et al. Effect of captopril on mortality and morbidity in patients with left ventricular dysfunction after myocardial infarction. $\mathrm{N}$ Engl J Med 1992;327:669-77.

4. The SOLVD investigators. Effect of enalapril on survival in patients with reduced left ventricular ejection fractions and congestive heart failure. N Engl J Med. 1991;325:293-302.

5. The Acute Infarction Ramipril Efficacy (AIRE) Study investigators. Effect of ramipril on mortality and morbidity of survivors of acute myocardial infarction with clinical evidence of heart failure. Lancet 1993;342:821-8.

6. Torp-Pedersen C, Kober L, TRACE Study Group. Effect of ACE inhibitor trandolapril on life expectancy of patients with reduced left-ventricular function after acute myocardial infarction. Lancet 1999;354:9-12.

7. Dagenais GR, Pogue J, Fox K, Simoons ML, Yusuf S. Angiotensin-converting-enzyme inhibitors in stable vascular disease without left ventricular systolic dysfunction or heart failure: a combined analysis of three trials. Lancet 2006;368 (9535):581-8. Review.

8. The Heart Outcomes Prevention Evaluation Study investigators. Effects of an angiotensin-converting enzyme inhibitor, ramipril, on cardiovascular events in high risk patients. N Engl J Med 2000;342:145-53.

9. The European Trial on Reduction of Cardiac Events with Perindopril in Stable Coronary Artery Disease investigators. Efficacy of perindopril in reduction of cardiovascular events among patients with stable coronary artery disease: randomised, double-blind, placebo-controlled, multicentre trial (the EUROPA study). Lancet 2003;362:782-8.

10. Braunwald E, Domanski MJ, Fowler SE, PEACE Trial investigators. Angiotensin-converting-enzyme inhibition in stable coronary artery disease. N Engl J Med 2004;351: 2058-68.

11. Remme WJ, Deckers JW, Fox KM, Ferrari R, Bertrand M, Simoons ML for the EUROPA Investigators. Secondary prevention of coronary disease with ACE inhibition-Does blood pressure reduction with perindopril explain the benefits in EUROPA? Cardiovasc Drugs Ther. 2009;23: This issue.
12. Schmieder RE, Hilgers KF, Schlaich MP, Schmidt BM. Reninangiotensin system and cardiovascular risk. Lancet 2007;369 (9568):1208-19.

13. Boring L, Gosling J, Cleary M, Charo IF. Decreased lesion formation in CCR2 $-/-$ mice reveals a role for chemokines in the initiation of atherosclerosis. Nature 1998;394:894-9.

14. Wassmann S, Stumpf M, Strehlow K, et al. Interleukin-6 induces oxidative stress and endothelial dysfunction by overexpression of the angiotensin II type 1 receptor. Circ Res 2004;94:534-41.

15. Griendling KK, Alexander RW. Oxidative stress and cardiovascular disease. Circulation 1997;96:3264-5.

16. Mancini GB, Henry GC, Macaya C, et al. Angiotensin-converting enzyme inhibition with quinapril improves endothelial vasomotor dysfunction in patients with coronary artery disease. The TREND (Trial on Reversing ENdothelial Dysfunction) Study. Circulation 1996;94:258-65.

17. Antony I, Lerebours G, Nitenberg A. Angiotensin-converting enzyme inhibition restores flow-dependent and cold pressor testinduced dilations in coronary arteries of hypertensive patients. Circulation 1996;94:3115-22.

18. Fennessy PA, Campbell JH, Mendelsohn FA, Campbell GR. Angiotensin-converting enzyme inhibitors and atherosclerosis: relevance of animal models to human disease. Clin Exp Pharmacol Physiol 1996;23:530-2.

19. Matsumoto T, Minai K, Horie H, Ohira N, Takashima H, Tarutani $\mathrm{Y}$, et al. Angiotensin-converting enzyme inhibition but not angiotensin II type 1 receptor antagonism augments coronary release of tissue plasminogen activator in hypertensive patients. $\mathrm{J}$ Am Coll Cardiol 2003;41:1373-9.

20. Ghiadoni L, Magagna A, Versari D, Kardasz I, Huang Y, Taddei $\mathrm{S}$, et al. Different effect of antihypertensive drugs on conduit artery endothelial function. Hypertension 2003;41:1281-6.

21. Voors AA, van Veldhuisen DJ, van Gilst WH. The current role of ACE inhibitors for secondary prevention in cardiovascular disease; from pathogenesis to clinical practice. Cardiovasc Drugs Ther 2006;20:69-73.

22. Deckers JW, Goedhart DM, Boersma E, et al. Treatment benefit by perindopril in patients with stable coronary artery disease at different levels of risk. Eur Heart J 2006;27:796-801.

23. The ONTARGET Investigators. Telmisartan, ramipril, or both in patients at high risk for vascular events. $\mathrm{N}$ Engl J Med 2008;358:1547-5.

24. Pfeffer MA, McMurray JJ, Velazquez EJ, et al. Valsartan, captopril, or both in myocardial infarction complicated by heart failure, left ventricular dysfunction, or both. N Engl J Med 2003;349:1893-906. Erratum, N Engl J Med 2004;350:203. 